\title{
Muscular Dystrophy
}

National Cancer Institute

\section{Source}

National Cancer Institute. Muscular Dystrophy. NCI Thesaurus. Code C84910.

A group of inherited progressive muscle disorders characterized by muscle weakness and eventual death of the muscle tissues. Examples include Duchenne muscular dystrophy, Becker's muscular dystrophy, Emery-Dreifuss muscular dystrophy, facioscapulohumeral muscular dystrophy, and limb-girdle muscular dystrophy. 